\title{
L'archéologie russe en Asie centrale en situation coloniale : quelques approches
}

\section{Svetlana Gorshenina}

Traducteur : Mathilde Reichler Imperiali

\section{OpenEdition \\ Journals}

Édition électronique

URL : http://journals.openedition.org/edl/354

DOI : $10.4000 /$ edl.354

ISSN : 2296-5084

\section{Éditeur}

Université de Lausanne

\section{Édition imprimée}

Date de publication : 15 mai 2012

Pagination : 183-222

ISBN : 978-2-940331-27-7

ISSN : 0014-2026

\section{Référence électronique}

Svetlana Gorshenina, "L'archéologie russe en Asie centrale en situation coloniale : quelques approches », Études de lettres [En ligne], 1-2 | 2012, mis en ligne le 15 mai 2015, consulté le 19 décembre 2020. URL : http://journals.openedition.org/edl/354; DOI : https://doi.org/10.4000/edl.354 


\section{L'ARCHÉOLOGIE RUSSE EN ASIE CENTRALE EN SITUATION COLONIALE: QUELQUES APPROCHES}

Le présent article examine les approches possibles pour une analyse de l'histoire de l'archéologie russe en Asie centrale, notamment dans le contexte colonial qu'a vécu le Turkestan au tournant des XIX et $\mathrm{XX}^{\mathrm{e}}$ siècles. L'étude met en évidence les traits spécifiques de ce qu'est une archéologie en situation coloniale et qui transparaissent à travers diverses pratiques dans l'organisation des recherches scientifiques.

\section{Introduction}

La publication, en 1978 à New York, du livre d'Edward W. Saïd, L'orientalisme. L'Orient créé par l'Occident ${ }^{1}$, a ouvert la voie à un examen critique des interactions fondamentalement ambiguës qui s'exercent entre le pouvoir et le processus de construction des savoirs en situation coloniale ${ }^{2}$. En partant du domaine de la littérature comparée, les méthodes d'analyse élaborées durant ces trois décennies au gré de violentes polémiques entre partisans et adversaires des différents courants des Postcolonial Studies ont vu leur champ d'application s'étendre

I. E. W. Saïd, Orientalism.

2. Voir, par exemple, pour le monde francophone les études portant sur la construction des savoirs dans une situation coloniale: E. Sibeud, Une science impériale pour l'Afrique? et "Les sciences sociales à l'épreuve de la situation coloniale»; S. Dulucq, C. Zytnicki (dir.), Décoloniser l'histoire?; J.-F. Klein, M.-A. de Suremain, "Clio et les colonies»; P. Singaravélou, "Le moment "impérial" de l'histoire des sciences sociales (1880-1910)». 
aux domaines des anthropologues, des historiens et des géographes ${ }^{3}$. L'acceptation de ces méthodes est cependant loin d'être homogène tant du point de vue spatial que disciplinaire.

D'un côté, certains chercheurs continuent de camper sur des positions de non-reconnaissance - partielle ou totale - des outils d'analyse postcoloniaux, comme on peut l'observer, par exemple, à une échelle importante en France ou dans les pays de la Communauté des Etats Indépendants (CEI), à commencer par la Russie. Dans le cas français, les principaux arguments du rejet reposent sur l'idée que la théorie d'Edward Saïd remonte à la philosophie française des années 1950-1960 et dénoncent le soi-disant simplisme des diverses ramifications de ce courant postcolonial (ce dernier argument est partagé par certains détracteurs de Saïd issus du monde anglophone) ${ }^{4}$. Dans le cas russe, c'est la particularité - sortant du commun - de l'orientalisme russo-soviétique et, de manière plus générale, de la particularité de la Russie/URSS elle-même que l'on avance souvent comme thèse de base pour exclure d'emblée toute possibilité d'appliquer les méthodes postcoloniales à l'analyse de leur histoire ${ }^{5}$.

D’un autre côté, quelques rares disciplines - essentiellement celles qui œuvrent sur les périodes anciennes - sont restées à l'écart, pratiquement jusqu'au milieu des années 1980, de l'examen des «sciences coloniales» considérées comme telles ${ }^{6}$. Prise dans cette optique, l'archéologie, qui

3. Voir l'introduction de M.-C. Smouts, «Le postcolonial, pour quoi faire», qui distingue quatre grands ensembles dans les Postcolonial Studies comme «la littérature comparée postcoloniale», "la déconstruction du discours colonial», "les Subaltern Studies» et "les “lectures postcoloniales" de la mondialisation»: M.-C. Smouts (éd.), La situation postcoloniale. Pour une bonne analyse des différents courants des Post-Studies voir: J. Pouchepadass, "Que reste-t-il des Subaltern Studies?» et "Le projet critique des postcolonial studies entre hier et demain». Voir également un autre bilan historique des Postcolonial Studies proposé par D. Kennedy, «Imperial History and Post-Colonial Theory".

4. J.-F. Bayart, Les études postcoloniales, p. 9-12 et 20-39 (sur les sources des Postcolonial Studies), passim (son point de vue a été à son tour critiqué dans un dossier spécial «Racial France» de la revue Public Culture, J. Roitman (ed.), p. 85-231); E. Sibeud, "Post-Colonial et Colonial Studies», p. 90. Pour une critique anglophone voir également R. Irwin, For Lust of Knowing.

5. Voir en particulier le manuel publié par l'Université de Saint-Pétersbourg: E. I. Zelenev, V. B. Kasevič, Vvedenie v vostokovedenie, p. 25.

6. P. Bourdieu, «Les conditions sociales de la production sociologique»; E. W. Saïd, Orientalism et Culture and Imperialism; D. Nordman, J.-P. Raison (éds), Sciences de l'homme et conquête coloniale; P. Petitjean (éd.), Les sciences hors d'Occident au XX'e siècle. 
se voit romantisée et exotisée dans tous les contextes extranationaux, est présentée souvent comme faisant partie du domaine de la «connaissance pure», puisqu'elle est censée être préservée des spéculations impérialistes et affranchie de toute mission d'ordre pratique en relation avec l'assujettissement colonial d'une nation à une autre. Définie dans le cadre de la notion d'internalizm, cette particularité des études relatives à l'histoire de l'archéologie a longtemps consisté dans l'élaboration d'une trame chronologique des découvertes et dans la vénération de personnalités marquantes - de "maîtres" - hors de toute mise en perspective historique. Cette approche contraste avec les innombrables analyses faites des rapports entre archéologie et nationalisme (pourtant souvent directement lié à l'impérialisme) et dans lesquelles l'archéologie est décrite comme une discipline politiquement engagée, voire un outil idéologique ${ }^{7}$. Elle a été de ce fait mise à mal dès les premières critiques formulées à l'égard de l'histoire traditionnelle de l'archéologie, au point que cette dernière s'est vue qualifier dans sa totalité comme un discours colonial ${ }^{8}$.

Dans ce contexte, le choix du présent thème, l'archéologie russe en Asie centrale en situation coloniale, a posé d'emblée plusieurs problèmes méthodologiques, à commencer par la question d'ordre général qui est de savoir si l'on peut parler d'une "archéologie coloniale» en tant que telle, avant de pouvoir s'interroger sur un cas particulier, celui de l'expérience russe au Turkestan, à partir du point de vue proposé.

7. J. A. Atkinson, I. Bank, J. O'Sullivan (eds), Nationalism and Archaeology; M. Díaz-Andreu, T. Champion (eds), Nationalism and Archaeology in Europe; Ph. L. Kohl, C. Fawcett (eds), Nationalism, Politics, and the Practice of Archaeology. Pour un compte-rendu critique portant sur cet engouement pour les rapports entre archéologie et nationalisme, voir M.-A. Kaeser, «Nationalisme et archéologie». Dans le monde russo-soviétique cette problématique a été également largement défrichée, notamment par V. A. Shnirel'man; voir en dernier lieu A. E. Petrov, V. A. Šnirel'man, Falsifikacija istoričeskikh istočnikov i konstruirovanie ètnokratičeskikh mifov.

8. Pour un aperçu de ce courant critique voir O. Moro-Abadía, "The History of Archaeology as a "Colonial Discourse" ", p. 4 sq., puis sa démonstration qualifiant l'histoire traditionnelle de l'archéologie comme un discours colonial, p. 7-14. 
1. Des "Archéologie(s) coloniale(s)», "colonialiste(s)" ou "en situation coloniale»

L'examen des liens entre l'archéologie et le colonialisme est pourtant loin de constituer un scoop. Esquissée pour la première fois par Bruce Trigger il y a plus d'un quart de siècle ${ }^{9}$, cette problématique a déjà attiré l'attention de nombreux chercheurs malgré une ambiguïté terminologique qui est loin d'être anodine. En effet, prise telle quelle, l'expression «archéologie coloniale" se prête à des interprétations très larges, qui englobent non seulement les activités archéologiques en situation coloniale, mais aussi l'acception héritée du domaine des études classiques, dont le but est de retracer l'histoire du colonialisme tel qu'il s'est propagé dans le monde gréco-romain, ou d'analyser l'histoire des sociétés colonialistes des XVI ${ }^{\mathrm{e}}$-XIX ${ }^{\mathrm{e}}$ siècles. Dans le cas de l'Antiquité, la problématique coloniale est destinée, entre autres, à «démontrer les possibilités d'une contribution archéologique à la compréhension du colonialisme» en épaulant l'anthropologie ${ }^{10}$, ou à démêler le mécanisme des "contacts culturels" ${ }^{11}$. Dans le second cas, l'objet des études peut être représenté par des sociétés de colons modernes, notamment aux Amériques ${ }^{12}$ ou en Océanie ${ }^{13}$.

L'emprise de l'archéologie classique est telle que, même si des démarches initiales théoriques tentent d'adopter le regard critique de l'analyse épistémologique en mettant en question la base même de cette discipline, il n'est pas rare de voir les propos tenus prendre ensuite une couleur plus traditionnelle. C'est, par exemple, le cas pour l'Archaeology of Colonialism, édité en 2002 par Claire L. Lyons et John Papadopoulos, où la problématique postcoloniale d'une science idéologisée a été effectivement évoquée de manière synthétique dans l'introduction ${ }^{14}$, sans toutefois se refléter dans le cœur du volume puisque les articles publiés

9. B. G. Trigger, "Alternative archaeologies», p. 360-363.

Io. M. Dietler, "L'Archéologie du colonialisme», p. 135 et Archaeologies of Colonialism.

II. S. W. Silliman, "Culture Contact or Colonialism?".

I2. G. Waselkov, The Archaeology of French Colonial North America; K. G. Kelly, M. D. Hardy (eds), French Colonial Archaeology in the Southeast and Caribbean.

I3. Voir les travaux, définis en tant qu' «archéologie coloniale», menés par l'Institut d'archéologie de la Nouvelle-Calédonie et du Pacifique, et dont un des sujets concerne l'étude des bagnes.

I4. C. L. Lyons, J. K. Papadopoulos, The Archeology of Colonialism. 
accordent leur préférence à l'archéologie des colons plutôt qu'à l'analyse de l'archéologie en situation coloniale. Ces deux archéologies ont été également réunies dans le cadre d'une étude réalisée par Chris Gosden sur la longue durée ${ }^{15}$.

Rendue difficilement utilisable par la contamination des deux approches évoquées ci-dessus, l'expression "archéologie coloniale» s'oppose en un fort contraste à celle $\mathrm{d}^{\prime}$ ' archéologie colonialiste» (colonialist archaelogy) inventée par Trigger ${ }^{16}$, qui traduit de manière trop linéaire et sans aucune ambiguïté l'état d'une discipline scientifique qui, dans le couple pouvoir-savoirs, serait définitivement soumise, parfois de manière volontaire ${ }^{17}$, à des enjeux idéologiques ou géopolitiques. De ce fait, cette expression a encore plus de mal à trouver sa place dans le vocabulaire des chercheurs, toujours méfiants lorsqu'il s'agit de simplifier l'analyse de la production des connaissances et soucieux d'éviter tout recours à des jugements de valeur ${ }^{18}$.

En revanche, l'expression "archéologie en situation coloniale" semble moins réductrice, dans la mesure où elle pourrait correspondre aux démarches scientifiques entreprises dans un contexte colonial avec des particularités comme celles qui ont été définies par Georges Balandier ${ }^{19}$, soit pour répondre de manière consciente aux attentes du monde politique, soit en reflétant, à leur insu, les dogmes idéologiques de l'époque.

Malgré ces ambiguïtés terminologiques persistantes, les approches postcoloniales à l'égard de l'archéologie comprise dans le cadre du worldsystem ont trouvé un terrain favorable dans le monde essentiellement

15. Ch. Gosden, Archaeology and Colonialism.

I6. B. G. Trigger, «Alternative archaeologies».

17. De ce point de vue, si l'on veut mettre en évidence un exemple de collaboration délibérée entre des chercheurs et un régime politique, on peut signaler le cas de l'archéologie nazie: J.-P. Legendre, L. Olivier, B. Schnitzler (éds), L’archéologie nationalesocialiste dans les pays occupés à l'ouest $d u$ Reich.

I8. Pour une critique voir, entre autres, N. Schlanger, «Identités recomposées» et "Archéologie coloniale et identité nationale en Afrique australe».

19. L'expression "situation coloniale" tirée de l'intitulé d'un article de Georges Balandier ("La situation coloniale: approche théorique»), paru en 1951, est devenue une formule-clé pour la compréhension du phénomène colonial: S. Dulucq, J.-F. Klein, B. Stora (éds), Les mots de la colonisation, p. 106. Le cinquantenaire de son article a été célébré à New York en 2002: E. Saada (éd.), Regards croisés. 
anglo-saxon ${ }^{20}$. A partir de divers cas de figure allant de l'Inde à l'Afrique, ces analyses s'approprient actuellement d'autres terrains, moins "traditionnels" pour les études postcoloniales (Israël, Xinjiang), comme en témoigne récemment le catalogue d'exposition Das grosse Spiel: Archäologie und Politik zur Zeit des Kolonialismus (1860-1940) ${ }^{21}$. L'intérêt grandissant pour cet aspect de l'histoire de l'archéologie transparaît également à travers les soutenances récentes de plusieurs thèses de doctorat ${ }^{22}$ étroitement liées aux problématiques plus larges du patrimoine ${ }^{23}$, du développement et de l'exercice de l'archéologie occidentale à l'étranger ${ }^{24}$.

S'alignant sur ce courant de pensée qui tend à revoir le schéma de la constitution des savoirs par rapport aux régions non occidentales ${ }^{25}$, un colloque a été organisé les 22 et 23 mars 2011 par l'équipe du CNRS Réseau Asie - IMASIE ${ }^{26}$ sous le titre «Archéologie(s) coloniale(s) : une

20. M. Díaz-Andreu, A World History of Nineteenth-Century Archaeology, p. 209317; M. Given, The Archaeology of the Colonized; H. I. Pai, Constructing "Korean" Origins; H. P. Ray, Colonial Archaeology in South Asia; N. Shepherd, "Heading South, Looking North»; N. Schlanger, «Identités recomposées».

2I. Ch. Trümpler (Hrsg.), Das grosse Spiel; voir notamment dans cet ouvrage l'importante bibliographie consacrée à l'archéologie et au colonialisme (p. 633-639).

22. Cf. L. Gillot, La mise en valeur des sites archéologiques; C. Gutron, L’archéologie en Tunisie; A. Nanta, Débats sur les origines du peuplement de l'archipel japonais dans l'anthropologie et l'archéologie; I. Simoni, Archeologia coloniale in Libia.

23. M. Volait, Fous du Caire; I. Maffi, Pratiques du patrimoine et politiques de la mémoire en Jordanie; N. Oulebsir, Les usages du patrimoine.

24. Par rapport à l'archéologie française à l'étranger voir, par exemple, E. GranAymerich, "L'archéologie française à l'étranger" (exemple de l'histoire traditionnelle de l'archéologie); F. Olivier-Utard, Politique et archéologie; N. Chevalier, La recherche archéologique française au Moyen-Orient.

25. Voir, entre autres, le colloque La préhistoire des Autres. Comment l'archéologie et l'anthropologie abordent le passé des sociétés non occidentales, les 18 et 19 janvier 2011, Musée du Quai Branly/INRAP.

26. Ce colloque a été organisé par Alexandra Galitzine-Loumpet, Svetlana Gorshenina et Claude Rapin (voir le programme: http://www.reseau-asie.com/media3/ conferences-debats/archeologie-coloniale-journees-d-etudes-1/). Podcast de l'émission spéciale consacrée au colloque dans le cadre de l'émission "Salon noir» de Vincent Charpentier, France Culture, le 13 avril 2011 : http://www.inrap.fr/archeologie-preventive/Ressources-multimedias/Emissions-de-radio/Le-Salon-noir/Radio-Le-Salonnoir/p-12954-Autour-des-archeologies-coloniales.htm. Pour les résultats du colloque voir les Nouvelles de l'archéologie ( $\mathrm{n}^{\circ} 126$, décembre 2011 ; une seconde partie paraîtra dans la même revue en juin 2012). Pour la possibilité même d'analyser l'archéologie russo-soviétique en Asie centrale à partir du point de vue d'une situation coloniale voir 
approche transversale", afin de pouvoir mieux approfondir cette problématique encore à peine défrichée et extrêmement sensible, et de savoir si la catégorie de l'«archéologie en situation coloniale» reste bonne à penser pour toutes les aires culturelles.

Lorigine des débats se situe dans le constat, exposé dans l'argumentaire du colloque, que "l'archéologie est née en Europe comme discipline au service de la genèse de la Nation, à une époque marquée par la croissance de la compétition coloniale». Cette double particularité des XVIII et XIX ${ }^{\mathrm{e}}$ siècles, qui ont été à la fois l'époque de la cristallisation de la plupart des nations européennes ${ }^{27}$ et celle de la formation des possessions coloniales (il faut comprendre ici la "deuxième phase» du colonialisme européen ${ }^{28}$ ), a entraîné l'apparition de deux «types" d'archéologie. La première serait celle qui s'est développée en Europe sur le terrain «national» ou que l'on a perçu comme tel même au-delà des frontières nationales, en lien direct avec les mythes fondateurs de l'identité européenne, notamment marquée par l'exaltation de la culture gréco-romaine ${ }^{29}$; la seconde serait une archéologie dont le territoire d'investigation a été déterminé par les lointaines possessions coloniales. Si, dans le premier cas, l'archéologie, appelée à construire le passé «national» des Nations, a été dans une large mesure liée à la création des mythes "nationaux" européens, quelle a été sa spécificité dans le second cas, et comment faudrait-il qualifier l'archéologie qui s'est développée dans ce contexte

S. Gorshenina, C. Rapin, «De l'archéologie russo-soviétique en situation coloniale à l'archéologie post-coloniale en Asie centrale» (certains éléments de ce travail sont reproduits dans le présent article).

27. A.-M. Thiesse, La création des identités nationales.

28. S. Dulucq, J.-F. Klein, B. Stora (éds), Les mots de la colonisation, p. 4.

29. Cet engouement pour la civilisation gréco-romaine, vue comme source directe de la civilisation européenne, explique la création des instituts européens en Grèce, en Italie et même en Turquie. L'archéologie "classique» développée dans ce cadre par des chercheurs sortant essentiellement de classes sociales élevées a été vue comme une discipline destinée à fournir des éléments indispensables à l'établissement de l'histoire européenne. Dans ce contexte, le développement des archéologies purement "nationales", représentées essentiellement par la protohistoire et exercées par des protagonistes appartenant souvent aux classes moyennes, a pris du retard: le mythe des ancêtres celtiques, perçus comme «sauvages» par rapport aux Grecs ou aux autres peuples hellénisés « raffinés", a initialement exercé une attraction très faible jusqu'à l'arrivée sur la scène intellectuelle du romantisme allemand avec sa glorification des "forces barbares». Pour une analyse de trajectoires des archéologies «classique» et "nationales», voir J.-P. Demoule, Ch. Landes (dir.), La fabrique de l'archéologie en France. 
extranational? Est-il possible d'affirmer qu'une situation coloniale a pu "colorer" cette discipline en lui fournissant des modèles de réflexion et des structures spécifiques reflétant sa nature singulière? Et, partant, a-telle produit un type d'archéologie spécifique et facilement reconnaissable ou plusieurs types d'archéologie dans des situations coloniales distinctes?

Lors du colloque, cette question a été confrontée, à l'échelle du siècle (1860-1960), aux multiples exemples d'archéologies mises en place dans diverses situations coloniales, de l'Asie à l'Afrique et de l'Amérique au Pacifique. Cette approche comparative, adoptée de manière délibérée, a montré qu'il est en principe possible, sans nier les particularités de certains exemples, de discerner certains mécanismes épistémologiques, des pratiques organisationnelles (notamment la création de structures adaptées au contexte colonial) et l'émergence de nouveaux acteurs, amateurs ou professionnels, marqués par des comportements spécifiques découlant de l'emprise normative des systèmes de domination.

Les réflexions menées en commun ont montré dans quelle mesure l'objet des recherches archéologiques varie en fonction de la situation coloniale instaurée dans des territoires entièrement ou partiellement assujettis à une administration coloniale directe ou indirecte, ou dans le cadre d'un protectorat ou d'une forte influence politique. Le fait qu'un espace colonisé se situe géographiquement à proximité ou non de l'Europe, ou qu'il soit défini comme une aire disposant ou non d'un passé "européen ", a également prédéfini les investigations de l'archéologie en situation coloniale en agissant selon une dialectique de reconnaissance et de distanciation. Ainsi, les études archéologiques en Afrique du Nord, au Proche-Orient et en Asie centrale ont au début exclusivement porté sur le passé "antérieur " à celui des peuples soumis, se concentrant sur la recherche des origines de la civilisation européenne dans des sites définis comme gréco-romains et en omettant les monuments dits «indigènes». Placée dans ce cadre colonial, l'archéologie "classique» a été employée pour renforcer des mythes "nationaux» et a de ce fait estompé la ligne de démarcation entre ces deux types d'archéologie - "coloniale» et «nationale».

Dans d'autres circonstances et pour des régions géographiquement plus éloignées, des institutions comme l'Ecole française d'ExtrêmeOrient, l'Institut français d'Afrique noire ou The Royal Anthropological Institute de Grande-Bretagne ont choisi comme objet d'investigation le passé «national» des autres peuples, dont la découverte a mené à des 
situations contradictoires. Si, d'un côté, l'absence de résultats rapides s'est transformée en négation de l'existence d'une "véritable» histoire de l'Afrique jusqu'au début de la période coloniale ${ }^{30}$, d'un autre côté, la découverte de monuments importants d'un passé extra-européen, comme en Indochine, en Inde ou en Haute-Asie ${ }^{31}$, a ébranlé la civilisation européenne dans sa position centrale dominante. L'état déplorable des monuments des Autres a cependant été interprété comme l'indicateur d'une situation de décadence et de dégradation culturelle ${ }^{32}$, qui n'a ultérieurement pas empêché les ex-colonisés d'adapter les résultats de l'«archéologie en situation coloniale» à leurs discours nationalistes en faveur de l'indépendance et à un processus de réinvention des traditions ${ }^{33}$.

Le colloque a également permis de suggérer que certains des modèles étudiés peuvent être facilement définis pour des rapports avec l'«archéologie en situation coloniale», alors que d'autres, comme le cas russo-soviétique en particulier, exigent une description plus nuancée.

\section{L'application des catégories postcoloniales à l'histoire et à l'archéologie russo-soviétique}

Au départ, la formulation même de cette problématique peut sembler dépourvue de sens aux yeux des archéologues tant postsoviétiques qu’occidentaux, tant la vision des sciences de l'Antiquité de la Russie tsariste et surtout de l'URSS contraste avec les représentations de l'archéologie bâties par les puissances occidentales. Alors que, par exemple, l'imagetype de l'archéologue montré dans les manuels scolaires français des années cinquante-début soixante est celle de l'explorateur à casque colonial effectuant des fouilles dans des dépendances lointaines fortement exotisées (avec une végétation tropicale, des ruines pittoresques, des chameaux et des ouvriers en costumes traditionnels) ${ }^{34}$, la représentation

30. C. Coquery-Vidrovitch, "De la périodisation en histoire africaine».

31. A titre d'exemple, voir Ph. Forêt, La véritable histoire d'une montagne plus grande que l'Himalaya.

32. Voir, par exemple, les premières étapes de l'étude du stupa de Sañchi: T. GuhaThakurta, "Production et reproduction d'un monument".

33. E. Hobsbawm, T. Ranger, The Invention of Tradition.

34. A. Chante, «Images de l'archéologie dans les manuels scolaires». 
de l'archéologue diffusée dans la société russo-soviétique répond à un schéma complètement différent, où l'image de l'aventurier à l'Indiana Jones a cédé la place à la poétique du prolétaire stakhanoviste popularisée à l'époque soviétique ${ }^{35}$.

Cette différence dans les clichés peut être considérée comme le prologue d'une discussion sur le caractère de la présence russe en Asie centrale, dont les appréciations dans les études occidentales, russes et centrasiatiques restent jusqu'à nos jours très aléatoires. Si, dans les études occidentales, on qualifie traditionnellement, à quelques exceptions près, de coloniale la présence russe en Asie centrale, la position des chercheurs (post)soviétiques, tant russes que d'Asie centrale, diverge de manière cardinale, entre celle d'une condamnation violente de l'attitude colonialiste des pouvoirs russe et soviétique et celle d'une négation complète de la réalité coloniale dans les pratiques soviétiques. Cette divergence dans les appréciations dépend avant tout des schémas idéologiques qui ont successivement pris place dans la recherche au cours des XIX et $\mathrm{XXI}^{\mathrm{e}}$ siècles et reflète pour l'essentiel des réalités (géo)politiques ${ }^{36}$. Il convient surtout de souligner que la polarité et la couleur émotionnelle des approches ne doivent pas être liées à une prétendue singularité de l'histoire russo-soviétique, mais plutôt à l'extrême politisation

35. En Asie centrale, l'archéologie se voit alignée dès l'aube du régime soviétique sous le contrôle de structures étatiques situées aussi bien en métropole (voir la mission de Boris Denike [1885-1941] envoyée à Termez en 1926-1928 par le Musée des peuples de l'Orient de Moscou) que sur place (Turkomstaris-Sredazkomstaris-Uzkomstaris, années 1920-1930). Elle va aussitôt dépendre des grands travaux de modernisation exécutés selon les plans quinquennaux (voir, par exemple, le contrôle archéologique assuré par Mikhail Masson [1897-1986] en 1939 pendant la construction du grand canal du Ferghana) et sera appelée à fournir des résultats "applicables» (voir notamment le manifeste de Mikhail Masson pour une "archéologie appliquée» susceptible de guider des grands travaux d'irrigation, ainsi que le programme scientifique que la mission de Sergej Tolstov [1907-1976] au Khorezm a largement ciblé sur l'étude de l'Ouzboï, l'ancien lit de l'Amou-Daria). L'uniforme militaire massivement adopté entre les deux guerres par les archéologues soviétiques, officiellement qualifiés de prolétaires intellectuels, reflète la militarisation de la société soviétique, sans dévoiler le processus des répressions staliniennes responsable de nombreuses victimes dans ce milieu professionnel. Voir, en partie, S. Gorshenina, C. Rapin, Les archéologues en Asie centrale, p. 61-67.

36. Pour une analyse détaillée du caractère de la présence russe en Asie centrale à travers le prisme des débats historiographiques des XIX ${ }^{\mathrm{e}}-\mathrm{XXI}^{\mathrm{e}}$ siècles voir S. Gorshenina, «La marginalité du Turkestan colonial russe est-elle une fatalité, ou l'Asie centrale postsoviétique entrera-t-elle dans le champ des Post-Studies». 
qui a toujours accompagné l'étude de la problématique coloniale dans le contexte de l'Empire russo-soviétique.

Quand l'Empire russe absorbe l'Asie centrale au XIX' siècle, tous les acteurs, de l'Europe à l'Asie, sont conscients que l'on a affaire à un acte colonial. S'appuyant sur l'expérience coloniale européenne à laquelle les fonctionnaires tsaristes se sont référés à maintes reprises dans leurs projets et leurs réflexions, les théoriciens et les praticiens de l'aménagement colonial du Turkestan soutiennent uniquement l'idée que la conquête et la colonisation russes sont "meilleures» que celle de l'Europe occidentale, en raison de la position géographique spécifique de la Russie, de «son rôle missionnaire d'unification de l'Orient et de l'Occident» et des particularités du "caractère russe", considéré comme "plus ouvert» et "plus capable d'adaptation et d'assimilation " ${ }^{37}$.

A l'époque soviétique, le thème de la présence russe en Asie centrale pendant la période tsariste voit l'apparition de nouvelles lectures. La vision soviétique de ce problème va ainsi passer du concept du «mal absolu» des années 1920 (G. I. Safarov, M. N. Pokrovskij, T. R. Ryskulov, P. G. Galuzo, etc. ${ }^{38}$ ), à celui du "moindre mal» des années 1950 (M. V. Nečkina, A. V. Jakunin, I. S. Braginskij, S. Radžabov et V. A. Romodin), avant de terminer vers les années 1970 par celui d'un "bien indiscutable». Alors que les observateurs occidentaux de l'époque de la guerre froide ne remettent jamais en doute la nature répressive du pouvoir soviétique, les Soviétiques ne se remettent en revanche jamais eux-mêmes en question. Les mots "colonisation", "conquête/invasion militaire/coloniale», "colonie» disparaissent totalement de la terminologie employée pour être remplacés par les termes "intégration", «incorporation ", "rattachement" ou encore "question nationale», qui reflètent des approches nouvelles et qui permettent de renforcer l'image positive de l'URSS, symbole d'une "union libre et anticoloniale de peuples frères ».

Lorsque survient la chute de l'URSS, les nouveaux Etats indépendants se démarquent radicalement du point de vue régnant en Russie et vont amalgamer toute l'époque russe et soviétique en un seul bloc à dénoncer plus ou moins vigoureusement en tant que période de domination

37. A. Kuropatkin, «Zapiska zavedujuščego Aziatskoj čast' ju glavnogo štaba polkovnika A. N. Kuropatkina po afganskomu voprosu, 27 nojabrja 1878 g. ».

38. Voir par exemple P. G. Galuzo, Turkestan-kolonija. 
coloniale. Il faudra attendre 2005 environ pour que le discours commence à prendre un caractère plus relativiste. Cependant, il est aujourd'hui encore difficile en Asie centrale postsoviétique de mettre en scène une dramaturgie nationale qui dénonce la domination coloniale, tandis que les historiographies scolaires et académiques hésitent entre la revendication d'une continuité empruntant la matrice soviétique et l'idée d'une rupture, donc la nécessité d'un travail de mémoire sur le $\mathrm{XX}^{\mathrm{e}}$ siècle ${ }^{39}$.

Pourtant, les approches à l'égard de l'histoire russo-soviétique se sont largement renouvelées à partir de la dissolution de l'Union soviétique en 1991 grâce à l'ouverture des archives, à un accès exceptionnel au terrain et au développement d'échanges soutenus entre chercheurs postsoviétiques et occidentaux, qui ont permis de mettre en place des schèmes de réflexion nouveaux ${ }^{40}$. La problématique impériale du régime tsariste et celle, différente, de son successeur soviétique ont été assez bien balisées ces vingt dernières années, tout en se limitant néanmoins pour l'essentiel aux périphéries occidentales, au Caucase et à la Sibérie. Les recherches ont le plus souvent porté sur la question des nationalités et de la construction des identités à travers le prisme, tantôt théorique, tantôt pratique, des politiques linguistiques et religieuses, des classifications de populations lors des recensements, ou des processus d'indigénisation des élites ${ }^{41}$. La majeure partie de ces études n’a en revanche

39. M. Laruelle, "Le paradigme du colonialisme en Asie centrale postsoviétique». Pour une application des méthodes d'analyse postcoloniale à l'expérience soviétique et postsoviétique, voir en dernier lieu les tentatives faites lors de deux colloques récents: d'une part au colloque «Postcolonial Approaches to Postsocialist Experiences» organisé par Alexander Etkind (University of Cambridge) et Dirk Uffelmann (University of Passau) les 24-25 février 2012 au King's College, Cambridge; et d'autre part à la table ronde organisée par Edmund Herzig et Stephanie Cronin «Russian Orientalism to Soviet Iranology: The Persian-speaking world and its history through Russian eyes» le 30 novembre 2012 au St Antony's College, University of Oxford.

40. W. Sunderland, "What is Asia to Us?».

4I. Parmi les très nombreuses publications de la nouvelle vague postérieure à la Perestroïka, voir en particuler M. Bassin, "Geographies of Imperial Identity»; N. B. Breyfogle, Heretics and Colonizers; D. R. Brower, E. J. Lazzerini (eds), Russia's Orient; J. Burbank, M. von Hagen, A. Remnev (eds), Russian Empire; J. Cadiot, Le Laboratoire impérial; R. P. Geraci, Window on the East; F. Hirsch, Empire of Nations; A. Jersild, Orientalism and Empire; M. Laruelle, L'Idéologie eurasiste russe ou comment penser l'empire; M. Kimitaka (ed.), Imperiology; Y. Slezkine, Arctic mirrors; N. G. Suvorova (otv. red.), Aziatskaja Rossija; A. J. Rieber, A. Miller (eds), Imperial Rule; T. Uyama (ed.), Empire, Islam, and Politics in Central Eurasia. 
accordé qu'une place marginale à l'Asie centrale, tandis que la grille des lectures postcoloniales n'y est que rarement appliquée ${ }^{42}$ en dépit du fait que les dernières publications relatives à la nature de l'orientalisme russe ont tenté de mieux situer le cas russo-soviétique du point de vue des études postcoloniales ${ }^{43}$. En outre, la question de la rupture ou de la continuité entre les régimes tsariste et soviétique n'a pas encore pu être clairement résolue en raison du caractère très sensible de son fonds politique et social; les chercheurs occidentaux n'ont eux-mêmes pas échappé aux dogmes qui veulent faire de l'époque soviétique un cas si spécifique qu'il semble difficile de la soumettre à une analyse postcoloniale ${ }^{44}$.

De manière générale, en ce qui concerne l'archéologie centrasiatique proprement dite, on observe que les études occidentales récentes sur l'histoire de la construction des savoirs dans ce domaine (sans parler des questionnements sur le fait colonial) ne sont pas nombreuses en comparaison avec les publications proprement archéologiques (bibliographies analytiques comprises) ${ }^{45}$. La littérature soviétique et postsoviétique, qui aborde plus fréquemment l'historique des recherches archéologiques en Asie centrale ${ }^{46}$, reste en général au niveau de la reconstruction de détails positivistes, sans se soucier des questions d'ordre épistémologique; en d'autres termes, elle reste entièrement «internaliste». L'une des caractéristiques de tous les travaux, tant occidentaux que (post)soviétiques, est leur subordination aux frontières politiques d'aujourd'hui:

42. Par rapport à l'Asie centrale, voir plus précisément B. Babadžanov, Kokandskoe khanstvo; D. R. Brower, Turkestan and the Fate of the Russian Empire; D. Brower, "Imperial Russia and its Orient»; S. Gorshenina, S. Abashin (éds), Le Turkestan russe; A. Khalid, "Locating the (Post-)Colonial in Soviet History" et "Backwardness and the Quest for Civilization»; J. Sahadeo, Russian Colonial Society in Tashkent; W. Sunderland, Taming the Wild Field, Colonization and Empire on the Russian Steppe.

43. L. de Meaux, La Russie et la tentation de l'Orient; D. Schimmelpenninck van der Oye, Russian Orientalism; V. Tolz, "Russia's own Orient».

44. Voir les analyses intéressantes de D. Geyer, "Modern Imperialism?"; N. Pianciola, "Décoloniser l'Asie centrale?»; A. Khalid, "Between Empire and Revolution".

45. A. Fenet, Documents d'archéologie militante; S. Gorshenina, C. Rapin, Les archéologues en Asie centrale; F. Olivier-Utard, Politique et archéologie.

46. Voir par exemple M. E. Masson, «Kratkij očerk istorii izučenija Srednej Azii v arkheologičeskom otnošenii »; B. A. Litvinskij (otv. red.), Vostočnyj Turkestan v drevnosti $i$ rannem srednevekov'e et "Zakaspijskij kružok ljubitelej arkheologii i istorii"; V. S. Solov'jov, U istokov arkheologii Tadžikistana (1813-1917). 
les reconstitutions de l'histoire de l'archéologie russo-soviétique en Asie centrale se font sans le moindre parallèle avec les situations du Xinjiang ou de l'Afghanistan, alors même qu'une telle comparaison permettrait de mieux comprendre les particularités de la conduite des recherches scientifiques dans les dépendances directes et au sein de puissances nominalement libres ${ }^{47}$.

Finalement, force est de reconnaître que l'histoire de l'archéologie russo-soviétique en Asie centrale en situation coloniale n'a pas encore attiré l'attention des chercheurs, au point que la formulation de cette problématique d'investigations sonne étrangement aux oreilles de certains collègues et provoque un net sentiment de rejet ${ }^{48}$.

47. Voir la discussion suscitée par la tentative de comparaison entre les archéologies soviétique et occidentale en Asie centrale proposée par S. Gorshenina et C. Rapin, Les archéologues en Asie centrale: S. Gorshenina, C. Rapin, "Kak istorik i arkheolog prevratilis' v buržuaznykh propagandistov». Sur le plan des recherches historiques générales, l'approche comparative est là aussi plutôt rare: B. Eschment, H. Harder (eds), Looking at the Coloniser; A. S. Morrison, Russian Rule in Samarkand 1868-1910.

48. Dans la CEI, les rapports entre l'archéologie russo-soviétique et le colonialisme se sont pourtant déjà manifestés dans la polémique concernant la momie d'Ukok (République de l'Altaï) découverte en 1993. Lors du processus d'ethnicisation de l'histoire de cette république autonome, de violentes accusations de colonialisme ont été lancées par les activistes locaux à l'adresse des archéologues russes, en particulier Vjačeslav Molodin et Natal'ja Polos'mak, de l'Institut de recherches en Archéologie et ethnographie de Novosibirsk (D. Vertukhov, «Kolonial'naja arkheologija»). Le prétexte de ces attaques a été le refus des scientifiques de transférer la momie de Novosibirsk au Musée national de Gorno-Altaïsk, ainsi que l'interprétation proposée de voir dans cette trouvaille un témoignage représentatif plutôt de l'Altaï, que de la culture iranienne. Malgré le caractère nettement nationaliste de la polémique, les éléments soumis à critique sont à mettre clairement en relation avec le mécanisme d'une «archéologie en situation coloniale», surtout si l'on tient compte du parallèle fait par D. Vertukhov entre la culture iranienne et le «berceau du peuple russe», ce qui depuis le XIX ${ }^{\mathrm{e}}$ siècle suppose l'existence de racines aryennes (donc, iraniennes) chez les ancêtres des Russes modernes (voir infra). Ce type de polémique lancée à propos de monuments ou de vestiges désignés comme relevant du patrimoine national est caractéristique de l'époque postcoloniale. Il peut trouver de nombreux parallèles, entre autres, en Océanie où les Kanak, qui n’ont jamais été vus en tant qu'héritiers directs des créateurs des anciens monuments de leur territoire, ont récemment réussi à ethniciser leurs interprétations et imposer l'interdiction d'exportation des trouvailles: Ch. Sand, J. Bole, A.-J. Ouetcho, "Evolutions du discours archéologique sur 150 ans d'histoire coloniale et postcoloniale en NouvelleCalédonie». 


\section{L'archéologie russe en Asie centrale tsariste: quelques traits coloniaux}

S'il n'existe pas d' «archéologie coloniale type», ni de "puissances coloniales classiques", car tous les exemples diffèrent selon les situations ${ }^{49}$, on peut toutefois suggérer la présence de certains indices représentatifs des liens entre l'archéologie et la colonisation au niveau des pratiques, des structures et des concepts. En prenant ces derniers comme matrices, on tentera ici d'en saisir les reflets dans le cas du Turkestan russe.

En ce qui concerne l'époque tsariste (des années 1860 à 1917), on peut noter que le regard porté par les hommes politiques et les intellectuels russes sur le Turkestan et les khanats centrasiatiques relève à grands traits de l'orientalisme tel qu'il a été décrit par Edward Saïd, quand bien même les orientalistes russes (vostokovedy) ont souvent dénoncé l'attitude impérialiste de leurs collègues occidentaux ${ }^{50}$. La presse russe, qui ne se distingue guère en cela de la presse européenne, présente invariablement le Turkestan comme une périphérie attardée du "monde moderne». Pratiquement chaque volume du célèbre Recueil Turkestanais - constituant la chronique détaillée de la présence russe en Asie centrale entre 1868 et 1916 - décrit les populations locales comme des "barbares", justifiant ainsi l'offensive russe au nom des «bienfaits de la civilisation européenne" ${ }^{51}$. Les conquêtes coloniales produisent des formations discursives spécifiques - aussi bien littéraires que visuelles - que l'on retrouve dans la muséographie des expositions turkestanaises ${ }^{52}$, chez les premiers peintres russes ayant séjourné en Asie centrale (en particulier dans les travaux de Vasilij Vereščagin ${ }^{53}$ ), dans les pages des «romans turkestanais» (comme ceux de Nikolaj Karazin) ou dans de multiples récits de voyage, soit toutes sortes d'œuvres qui ne font que conforter cette thèse en l'étayant de nouveaux détails pittoresques et exotiques. La

49. J.-F. Klein, M.-A. de Suremain, "Clio et les colonies».

50. V. Tolz, "Russia's own Orient».

5I. V. Méjow [Mežov], Recueil du Turkestan comportant des livres et des articles sur l'Asie centrale en général et la province du Turkestan en particulier, p. II. Pour une analyse de cette bibliothèque coloniale, compilée par Mežov, voir S. Gorshenina, «Le Recueil Turkestanais de Mežov".

52. S. Gorshenina, "La construction d'une image "savante” du Turkestan russe lors des premières expositions "coloniales" dans l'empire».

53. D. Schimmelpenninck van der Oye, "Vasilij V. Vereshchagin's Canvases of Central Asian Conquest», p. 195-203. 
ségrégation, clairement apparente dans la structure de toutes les villes du Turkestan, divisées en quartiers russes et autochtones ${ }^{54}$, n'est pas étrangère non plus aux relations instaurées entre les colons et les habitants locaux malgré le fait que la rupture entre les deux ne soit pas aussi importante que dans les colonies des puissances occidentales.

De cette attitude naît la conviction que les Russes "éclairés» sont les seuls capables d'évaluer le patrimoine centrasiatique à sa juste valeur, et que, selon leur estimation, c'est à eux que revient la tâche d'écrire l'histoire - la plus ancienne avant tout - de la région, en y procédant notamment à des fouilles archéologiques. Alors que les Occidentaux les voient eux-mêmes souvent comme des extra-Européens, les Russes ont de leur côté déjà bien assimilé la science archéologique qui s'est forgée en Europe comme une discipline politiquement engagée, en l'adaptant aux besoins de leur empire qui entre alors dans l'ère de la modernisation et des valeurs de l'Etat-Nation. Tout en représentant un élément structurant des Etats-Nations, cette discipline a été par la suite transférée en Asie centrale pour aider à créer une dépendance coloniale directe comme celle du Turkestan russe ou à établir une zone d'influence politique indirecte comme cela a été le cas au Turkestan chinois (Xinjiang) ou, dans une moindre mesure, en Afghanistan (infra).

En effet, deux types d'approche ont été élaborés par les archéologues russes à l'égard de l'Asie centrale: premièrement par rapport à leurs propres colonies au Turkestan russe - donc "chez soi" - et deuxièmement par rapport aux espaces appartenant formellement à la Chine - «chez les Autres» - qui ont été considérés comme une zone d'expansion impérialiste possible du tsar, puis des Soviets ${ }^{55}$. La différence entre ces deux approches se fait déjà remarquer dans l'organisation de la recherche: si, dans le premier cas, la structuration institutionnelle passe par la création de sociétés savantes et de comités de sauvegarde du patrimoine, le lancement d'enseignements supérieurs et la cristallisation des structures académiques sur place, dans le second cas, elle reste limitée à des missions de courte ou de moyenne durée qui s'appuient sur les réseaux diplomatiques. Cette différence est également visible au niveau des discours de légitimation que l'on forge sur la base des interprétations

54. J. Sahadeo, Russian Colonial Society in Tashkent, 1865-1923.

55. S. Gorshenina, Asie centrale. 
de travaux archéologiques, de même qu'elle transparaît aussi à l'égard des rapports que l'archéologie russe maintient avec l'archéologie occidentale.

Dans ces deux cas de figure, l'archéologie est donc ici - comme partout dans le monde extra-européen - une «science importée» de l'Europe. Dans les années 1970, quand les écoles locales - nées de la politique dite d'«indigénisation" (korenizacija) - parviennent à obtenir une force véritable, cette caractéristique constitue pour les historiographes centrasiatiques une pierre d'achoppement, qui se transforme en véritable rejet à partir de l'indépendance de 1991, quand on commence au plus haut niveau à exiger la "découverte» d'un père fondateur local pour chacune des disciplines scientifiques. C'est ainsi que l'on se met alors à considérer les collectionneurs centrasiatiques du XIX ${ }^{\mathrm{e}}$ siècle comme les véritables précurseurs des archéologies nationales, en dépit du fait que l'apparition du goût pour les vestiges archéologiques dans le milieu des intellectuels et des marchands turkestanais ait été en grande partie liée aux commandes des collectionneurs russes et européens et aux pratiques d'organisation des expositions coloniales turkestanaises ${ }^{56}$.

Comme dans les autres territoires sous influence de type colonial, l'archéologie devient une chasse gardée. L'intégration d'une partie de l'Asie centrale dans l'Empire russe sous forme de Gouvernorats des steppes et du Turkestan et l'établissement du protectorat russe sur les territoires de Khiva et de Boukhara conduisent pratiquement à un monopole russe sur l'exploration archéologique de cette région. La durée des séjours des étrangers et la possibilité même de visiter le Turkestan, sans parler de l'autorisation de mener des fouilles, dépendent directement de la bonne volonté de l'administration russe qui empêche souvent les chercheurs occidentaux d'accéder au terrain. Pour toute la période tsariste, moins de vingt Occidentaux ont été dénombrés au Turkestan russe dans le cadre d'investigations archéologiques, pour une activité d'envergure d'ailleurs plutôt insignifiante. N'ayant pas la possibilité de mener sur place des travaux de longue durée, ces chercheurs se limitent, au fil des voyages, à rédiger de brèves descriptions des monuments; ou bien, comme cela a été le cas pour Charles-Eugène Ujfalvy (1842-1904, voyages en 1879-1880) et Edouard Blanc (1858-1923, voyage en 1893), l'activité de terrain ne dépasse pas l'ouverture de fouilles superficielles

56. S. Gorshenina, Private Collections of Russian Turkestan in the 2nd Half of the 19th and Early 20th Century, p. 81-83. 
d'un jour, menées un peu au hasard ${ }^{57}$. De manière générale, leurs notes sont souvent fragmentaires; elles comportent de nombreuses imprécisions sur la chronologie, la toponymie et l'onomastique et se rattachent plutôt au genre des descriptions de type touristique ${ }^{58}$. Certains, comme Léon de Beylié (1849-1910, voyage en 1888) se voient refuser toute autorisation d'effectuer des fouilles ou des prospections et réduisent leurs observations des monuments anciens à quelques mentions superficielles ${ }^{59}$. La mission américaine de Raphael Pumpelly (1837-1923) qui obtient l'autorisation de travailler sans trop d'obstacles à Annau en 19031904 constitue la seule exception dans cette situation de quasi-monopole russe (à l'époque soviétique il sera d'ailleurs total jusque dans les années 1980).

En même temps, les archéologues russes ne se privent pas d'aller faire des fouilles chez les "Autres». La participation des archéologues russes à la course aux antiquités, notamment pour les manuscrits anciens, se fait sur un pied d'égalité avec les autres puissances occidentales (comme la France, l'Allemagne ou la Suède) et démontre que, au même titre que ses concurrents géopolitiques, la Russie voit alors l'archéologie comme une des composantes du Great Game ${ }^{60}$ où les préoccupations scientifiques se trouvent mêlées à la raison d'Etat. Dans le cadre de l'Asie centrale, le Turkestan chinois est un espace libre qui va permettre une compétition scientifique entre les chercheurs des puissances occidentales et du Japon tout à fait comparable à celle de la géopolitique. Alors que la signature d'un traité international en 1902 à Hambourg est censée permettre de délimiter les zones d'investigations sur le terrain entre chercheurs occidentaux, Russes compris, les Allemands violent les accords pratiquement dès le lendemain et l'exploration au Xinjiang prend alors inévitablement

57. Ch.-E. de Ujfalvy de Mezö-Kövesd, Le Syr-Daria, p. 81 ; E. Blanc, «Notes de voyage en Asie centrale», p. 827.

58. S. Gorshenina, "De l'archéologie touristique à l'archéologie scientifique» et Explorateurs en Asie centrale.

59. S. Gorshenina, "Voyage de Léon de Beylié à Samarkand en été 1888 ».

6o. Le terme de Great Game a été inventé par le capitaine Arthur Conolly, mort en 1842 décapité par l'émir de Boukhara. Par la suite, l'expression reparaît chez l'historien militaire Sir John Kaye qui se trouve posséder des lettres de Conolly; elle obtiendra enfin une reconnaissance mondiale dans Kim, le roman de Rudyard Kipling (1901). Dans le contexte de la confrontation russo-britannique dans la seconde moitié du XIX et au début du XXe siècle, le Great Game représente la lutte pour le contrôle de l'Asie centrale. 
parmi les puissances le caractère d'une course aux antiquités ${ }^{61}$. Les archéologues russes participent comme ceux des autres pays à l'exportation des manuscrits du Xinjiang, sans toutefois se priver de critiquer les interventions peu respectueuses de leurs concurrents ${ }^{62}$.

Une même concurrence archéologique va se manifester également dans les autres régions stratégiques où s'entremêlent les intérêts géopolitiques des diverses puissances, particulièrement en Iran et en Afghanistan. Tout en s'occupant essentiellement des Indes, la GrandeBretagne, principale rivale de la Russie, n'oublie pas les régions frontières afghanes, du Pamir et du Turkestan chinois où elle expédie des agents qui sont également chargés de faire des observations archéologiques. L'Allemagne essaie de rattraper son retard dans la constitution des colonies en envoyant des chercheurs au Turkestan chinois ${ }^{63}$. Même si ses intérêts coloniaux l'attirent ailleurs, la France s'intéresse à l'Iran, au Turkestan chinois et, dans une certaine mesure, au Turkestan russe. Mais c'est surtout en Afghanistan que son impact sera le plus important. L'Italie, le Japon et les Etats-Unis ne parviendront à s'activer efficacement en Afghanistan que dans les années 1950-1980.

Parmi d'autres parallèles avec les "archéologies coloniales" occidentales, on peut également évoquer l'intervention dans ce domaine scientifique de militaires professionnels qui se présentent en tant qu'amateurs de l'archéologie locale. Ce sont eux en effet qui lancent les premières fouilles au Turkestan et collectent des antiquités, soit en profitant de leur position administrative, soit pour répondre à la demande de leurs supérieurs et des intellectuels de la métropole ${ }^{64}$.

Ce n'est pas un résultat du hasard si le premier site choisi pour des fouilles de longue haleine est celui de Samarcande (fig. 1). Selon

6I. P. Hopkirk, Foreign Devils on the Silk Road; I. F. Popova, «Russian Expeditions to Central Asia at the Turn of the 20th Century", p. 30.

62. Ch. Trümpler (Hrsg.), Das grosse Spiel.

63. Idem.

64. En 1873, le chef de l'okrug (région) du Zarafšan, le général-major Alexandre Abramov (1836-1886), a envoyé en mission à Afrasiab le chef de l'otdel (district) de Samarcande, le major Borzenkov, pour y mener des fouilles archéologiques. En 1883, sur l'ordre du général-gouverneur, Mikhail Černjaev (1828-1898), des fouilles ont été entreprises par un fonctionnaire préposé aux missions spéciales, Vsevolod Krestovskij (1840-1895, connu par ailleurs comme écrivain). Pour plus de détails voir S. Gorshenina, «Premiers pas des archéologues russes et français dans le Turkestan russe (1870-1890)». 


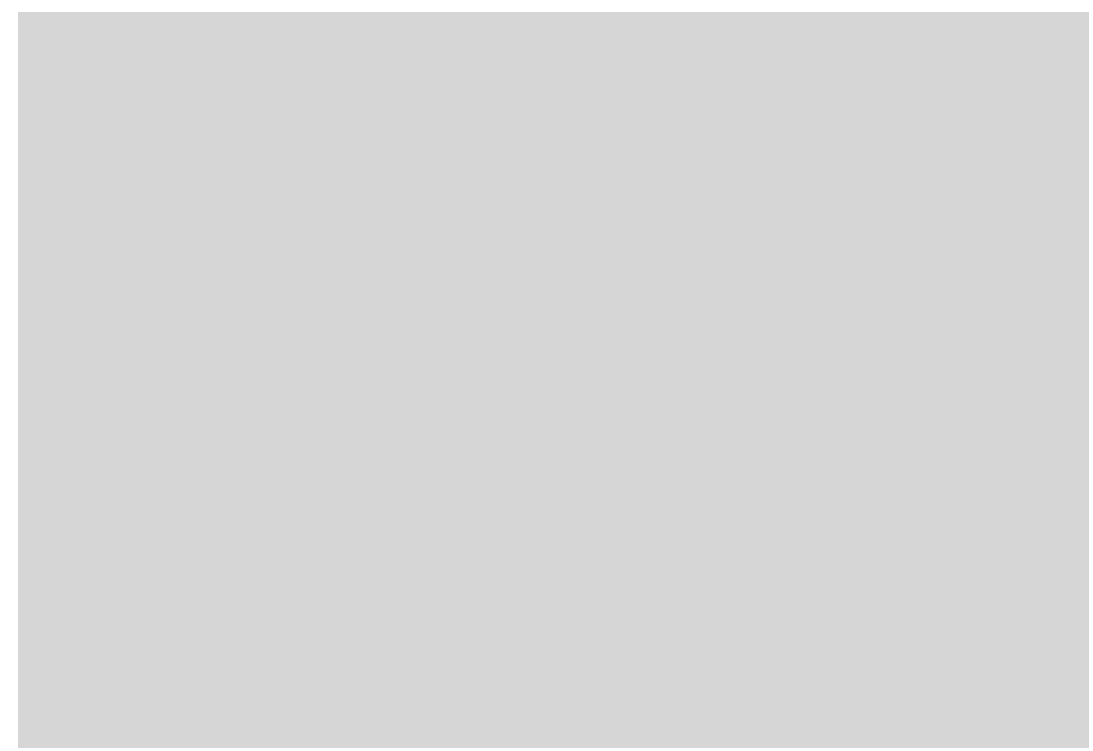

Fig. 1 - Vue de Samarcande. Photographie de V. A. Tresvjatskij, 1913-1915.

l'imaginaire collectif de l'époque, cette ancienne capitale de l'Empire timouride - et qui doit aussi beaucoup de sa gloire en Europe aux récits de Marco Polo - cache derrière ses façades islamiques quelques-uns des épisodes les plus connus de l'histoire antique que l'on considère comme le fondement de la civilisation européenne: dès le XVIII siècle, les travaux du géographe Jean-Baptiste Bourguignon d'Anville, "premier géographe du Roi de France», font pour la première fois le rapprochement entre la forme moderne Samarcande et Maracanda, une ville qui, pour les historiens gréco-romains et le géographe Claude Ptolémée, est liée à l'expédition d'Alexandre le Grand. Les sources écrites, qui narrent avec une relative précision le séjour de l'armée macédonienne dans la citadelle de Maracanda ${ }^{65}$, rapportent que c'est là que la garnison mise en place par Alexandre subit le siège du Sogdien Spitamène et que se serait déroulé le célèbre banquet au cours duquel Alexandre tua son compagnon de toujours Kléitos.

La recherche des traces du conquérant macédonien ne découle pas d'un simple effet de mode européenne (les Britanniques ont de leur côté également cherché des vestiges grecs en Inde, tandis que les Français se

65. Arrien 4.3.6-7, Quinte-Curce 7.6.10. 
sont lancés à la poursuite du mirage d'Alexandre en Afghanistan), mais reflète la quête identitaire des Russes qui, eux aussi, se revendiquent comme des Européens, héritiers des Grecs:

En apportant au fond de l'Asie l'influence et la graždanstvennost' européennes - écrit un journaliste anonyme exprimant un point de vue très répandu dans la Russie impériale -, nous exécutons une grande mission dont le premier apôtre a été Alexandre le Grand, en élargissant ainsi les frontières du monde civilisé ${ }^{66}$.

Divers raisonnements conduisent d'ailleurs les Russes à se chercher une origine aryenne en Asie centrale selon la même logique qui agite la recherche dans les autres pays occidentaux. Ce n'est donc pas un hasard si, en lançant la séance d'inauguration du Cercle Turkestanais des amateurs d'archéologie (Turkestanskij kružok ljubitelej arkheologii - TKLA) en 1895, Aleksandr Vrevskij (1834-1910), baron et général-gouverneur militaire du Turkestan, se félicite du fait que les Russes arrivent enfin en Asie centrale pour que la civilisation aryenne étincelle à nouveau dans sa proto-patrie:

A présent, le destin nous a conduits, nous, les Aryens, en ces lieux mêmes d'où sont autrefois partis nos ancêtres. Pour cette raison, nous avons le devoir sacré de réunir et de conserver les monuments historiques de ces contrées où, jadis, fleurissait une culture aryenne que nous sommes appelés à rétablir ${ }^{67}$.

Le présupposé - un parmi tant d'autres - selon lequel le «berceau aryen» se situe en Asie centrale est récupéré par les milieux politiques russes pour un discours qui va légitimer la conquête du Turkestan : comprise dans cette optique, l'expansion russe devrait perdre sa nature coloniale pour n'apparaître plus que comme un simple retour des Aryens dans leur patrie. Les premières manifestations de l'archéologie russe dans sa nouvelle colonie turkestanaise dévoilent cependant la nature bien coloniale du phénomène: le moteur des recherches étant fondé sur un discours politisé invoquant les «ancêtres aryens» des Russes, les activités du Cercle d'amateurs d'archéologie du Turkestan se concentrent dans une

66. Anonyme, «Po povodu vzjatija Samarkanda», p. 149.

67. Anonyme, «Discours du baron A. Vrevskij du 11 décembre 1895 prononcé lors de l'inauguration du Cercle». Traduction de M. Laruelle, "Le berceau aryen», p. 127. 
Fig. 2 - L'ossuaire zoroastrien de Bija-Najman découvert en 1908.

large mesure sur la quête de vestiges grecs, scythes ou chrétiens arméniens, nestoriens ou syriaques en Asie centrale, sur l'étude des langues iraniennes, des pétroglyphes et des inscriptions runiques, ainsi que sur les traditions «aryennes» zoroastriennes et bouddhistes (fig. 2).

Cette orientation du Cercle provoque une critique de Vasilij Bartol'd (1869-1930) qui regrette que la turcophobie générale régnant dans les cercles politiques russes touche également la science:

Une représentation sur-idéalisée des capacités culturelles des Aryens et de la barbarie des Türks ne pouvait pas ne pas influer sur l'appréhension des missions scientifiques de la Russie au Turkestan. Ainsi, en 1895, lors de l'ouverture d'un Cercle archéologique local sous la direction du pouvoir russe dans la zone, on a fait la proposition d'étudier la culture aryenne ancienne de la région, anéantie par les barbares turciques et connaissant son renouveau sous la domination d'autres Aryens, les Russes ${ }^{68}$.

68. Ibid., p. 123. 
Létude du "passé antérieur", défini comme aryen et lié au passé réel ou mythique de la Russie, apparaît ainsi plus importante que celle du passé «national» des peuples centrasiatiques. Cette tendance, qui domine dans les travaux des amateurs et historiens locaux, se manifeste également dans les réflexions des spécialistes de la capitale. Le Comité de la Commission archéologique impériale, qui dépêche en 1884 Nikolaj Veselovskij (1848-1918) avec la première expédition académique au Turkestan, n'évalue l'histoire de l'Asie centrale qu'à travers le prisme de l'histoire russe, sans perdre de vue les préoccupations de l'archéologie nationale dans la métropole: les instructions données indiquent qu'«en plus des fouilles des vestiges de villes, il serait opportun de faire des recherches également dans quelques kourganes, ce qui est important pour les fouilles de kourganes en Russie ${ }^{69}$.

Le particularisme de la situation coloniale en Russie se manifeste dans le fait que dans les milieux politiques et intellectuels russes la théorie «aryenne» peut cohabiter avec la thèse «touranienne», qui soutient une essence touranienne et non indo-européenne des Russes et présente ainsi ces derniers comme les héritiers directs de Gengis Khan. Paradoxalement, les applications politiques de ces idées produites par les vostočniki ${ }^{70}$ ne se distinguent pas de celles du discours "aryen»: sous cet angle, l'avancée des Russes vers le sud prend le caractère d'un retour aux sources nationales suivi d'une réunification avec les voisins de la patrie préhistorique ${ }^{71}$. La théorie touranienne n'empêche d'ailleurs pas de réaffirmer la nécessité de "civiliser" ces "Barbares d'Asiates" selon les usages européens. Dans le contexte archéologique, cette position idéologique se reflète, durant l'étude des monuments musulmans, dans les commentaires portés sur leur

69. V. S. Solov'jov, U istokov arkheologii Tadžikistana (1813-1917), p. 14.

70. Le courant de pensée russe, en réalité une branche de slavophiles, incarné notamment par Vasilij Vasil'ev (1818-1900), Fjodor Martens (1845-1909), Sergej Južakov (1849-1910), Èsper Ukhtomskij (1861-1921), Sergej Witte (1849-1915), privilégie des relations soutenues avec la Chine en projetant de construire un empire dont les frontières orientales vont au-delà de la grande muraille à l'image de son prototype, l'Empire de Gengis Khan, comme l'a décrit avec vigueur Nikolaj Trubeckoj (1890-1938). Selon Ukhtomskij, il est temps de rejeter l'héritage de Pierre le Grand et de revenir à l'état d'esprit propre à la Russie, qui est malheureusement complètement perdu au centre de l'empire, mais a survécu "au-delà de l'Altaï et le Pamir» (È. È. Ukhtomskij, Ksobytijam $v$ Kitae, t. 2, p. 2).

7I. M. Hauner, What is Asia to us?, p. 43-56; M. Laruelle, Mythe aryen et rêve impérial dans la Russie du XIX e siècle. 


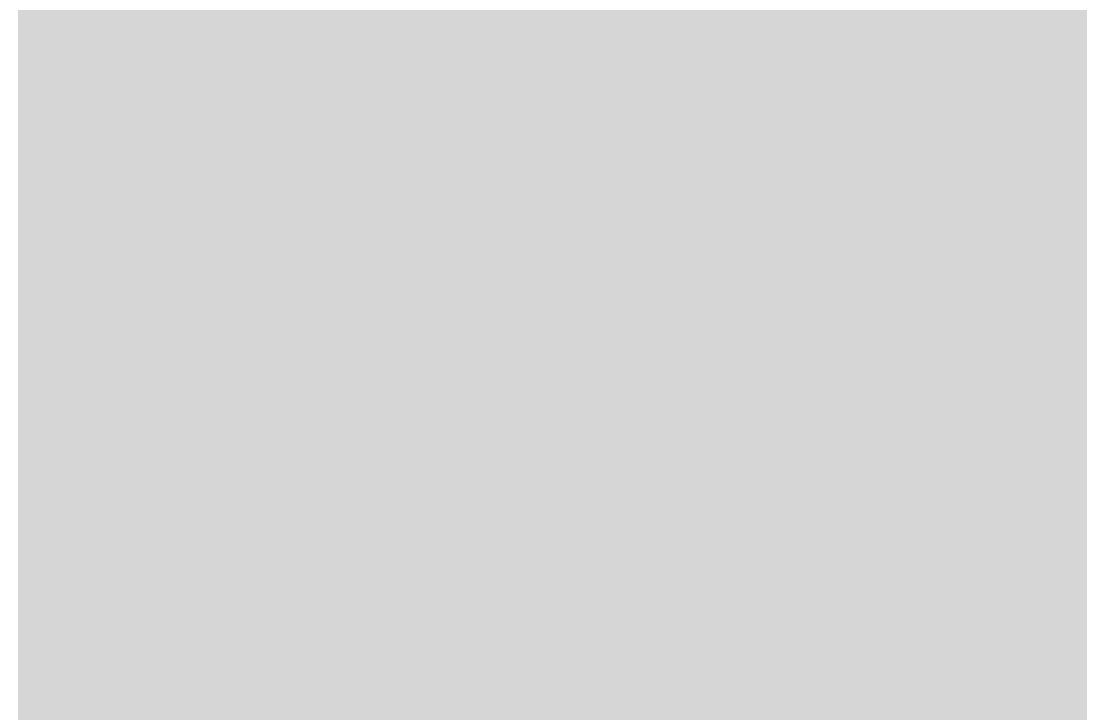

Fig. 3 - Les ruines du Vieux Merv.

mauvais état de conservation: les «ruines" des monuments turkestanais sont univoquement présentées comme des symboles de la décadence des civilisations centrasiatiques dont, selon l'expression courante à l'époque, «l'aspect même appelait les colonisateurs civilisés» à les «sauvegarder" immédiatement, légitimant ainsi la présence salvatrice russe (fig. 3).

L'ambiguïté de la situation s'amplifie encore durant les premières années du pouvoir soviétique, dans le cadre des premières démarches réalisées en Asie centrale russe dans le domaine de la patrimonialisation, notamment dans le cadre de l'activité du Comité des antiquités turkestanais (Turkomstaris); les membres du Comité s'attachent en effet à réconcilier plusieurs objectifs opposés comme la lutte lancée par les Soviets contre l'islam, le refus de poursuivre les pratiques tsaristes de préservation des monuments et faire face aux critiques que les Occidentaux pourraient émettre en cas de négligences dans le domaine de la défense du patrimoine islamique.

L'archéologie soviétique ne sera toutefois pas traitée dans la présente étude en raison du fait que le pouvoir soviétique qui l'a dirigée est très souvent considéré comme un cas extrêmement spécifique qui exige 
une analyse plus détaillée du fonctionnement de cette discipline ${ }^{72}$, notamment en ce qui concerne les actions affirmatives (ou de discrimination positive) lancées à l'égard des peuples «arriérés» (par la création d'Etats nationaux, l'éducation pour tous, l'accès à la médecine, la transformation des rapports de genre et des relations familiales, l'urbanisation, l'évolution des valeurs culturelles et religieuses, etc. ${ }^{73}$.

\section{En guise de conclusion}

L’analyse de l'archéologie russe au Turkestan dont la présente étude ne donne qu'une première esquisse permet de compléter la typologie des «archéologies en situation coloniale» tout en confrontant le cas russe aux expériences des autres puissances occidentales, notamment dans le domaine des mécanismes de la perception du passé de l'«Autre» extraeuropéen. Du statut des vestiges archéologiques et patrimoniaux à l'élaboration des mythes fondateurs des identités nationales des nouveaux pays centrasiatiques que s'approprient peu à peu les peuples de la région, la science archéologique russe en Asie centrale, et en particulier l'archéologie soviétique qui est restée pour le moment hors de notre analyse, a élaboré non seulement des axes de recherche déterminés, mais également des modèles normatifs de connaissance. Si l'on replace sa naissance dans le contexte colonial, l'histoire de cette archéologie pourrait donc aider à mieux comprendre les mécanismes de l'invention du passé de l'Asie centrale, que l'on évoque bien souvent comme l'une des "poudrières» du monde.

\section{Svetlana Gorshenina Réseau Asie-IMASIE, CNRS-FMSH, Paris}

Traduction des passages en russe de Mathilde Reichler Imperiali

72. Pour une brève analyse des périodes soviétique et post-soviétique voir S. Gorshenina, C. Rapin, «De l'archéologie russo-soviétique en situation coloniale à l'archéologie post-coloniale en Asie centrale».

73. T. D. Martin, The Affirmative Action Empire. 


\section{BIBLIOGRAPHIE}

Anonyme, "Discours du baron A. Vrevskij du 11 décembre 1895 prononcé lors de l'inauguration du Cercle", in Protokoly Turkestanskogo kružka ljubitelej arkheologii, Taškent, 1895.

Anonyme, "Po povodu vzjatija Samarkanda» ["A propos de la prise de Samarkand»], Birževye vedomosti [Journal officiel de la bourse], $\mathrm{n}^{\circ} 217$, in Turkestanskij sbornik, t. 8, s. d., p. 149.

Atrinson, John A., Bank, Iain, O'Sullivan, Jerry (eds), Nationalism and Archaeology. Scottish Archaeological Forum, Glasgow, Cruithne Press, 1996.

BABADŽAnov, Bakhtijar, Kokandskoe khanstvo: vlast', politika, religija [Le Khanat de Kokand: pouvoir, politique, religion], Tokyo/Taškent, 2010.

BALANDier, Georges, "La situation coloniale: approche théorique", Cahiers internationaux de sociologie, 11 (1951), p. 44-79.

Bassin, Mark, "Geographies of Imperial Identity", in The Cambridge History of Russia, vol. II : Imperial Russia, 1689-1917, ed. by Dominic Lieven, Cambridge, Cambridge University Press, 2006, p. 45-63.

BAYART, Jean-François, Les études postcoloniales. Un carnaval académique, Paris, Karthala, 2010.

Blanc, Edouard, "Notes de voyage en Asie centrale. Samarcande», Revue des Deux Mondes, 115 (1893), p. 796-837.

Bourdieu, Pierre, "Les conditions sociales de la production sociologique: sociologie coloniale et décolonisation de la sociologie», in Le mal de voir: ethnologie et orientalisme, politique et épistémologie, critique et autocritique: contributions aux colloques orientalisme, africanisme, américanisme (9-11 mai 1974), Ethnologie et politique au Maghreb (5 juin 1975), Paris, Union générale d'éditions, 1976, p. 416-426 (Cahiers de Jussieu 2).

Breyfogle, Nicholas B., Heretics and Colonizers: Forging Russia's Empire in the South Caucasus, Ithaca, Cornell University Press, 2005. 
Brower, Daniel, "Imperial Russia and its Orient: The renown of Nikolai Przhevalsky", The Russian Review, 53 (1994), p. 367-381.

Brower, Daniel R., Turkestan and the Fate of the Russian Empire, London, Routledge Curzon, 2003.

Brower, Daniel R., Lazzerini, Edward J. (eds), Russia's Orient. Imperial Borderlands and Peoples, 1700-1917, Bloomington/ Indianapolis, Indiana University Press, 2001.

Burbank, Jane, von Hagen, Mark, Remnev, Anatolyi (eds), Russian Empire: Space, People, Power, 1700-1930, Bloomington, Indiana University Press, 2007.

Cadiot, Juliette, Le Laboratoire impérial: Russie-URSS 1860-1940, Paris, CNRS-Editions, 2007.

Chante, Alain, «Images de l'archéologie dans les manuels scolaires», in L'archéologie et son image. Actes des VIII rencontres internationales d'archéologie et d'histoire d'Antibes, 29-31 octobre 1987, Juan-lesPins, Editions APDCA, 1988, p. 93-103.

Chevalier, Nicole, La recherche archéologique française au MoyenOrient, 1842-1947, Paris, Editions Recherche sur les civilisations, 2004 (Centre de recherche d'archéologie orientale 14).

Coquery-Vidrovitch, Catherine, "De la périodisation en histoire africaine. Peut-on l'envisager? A quoi sert-elle?», Afrique \& histoire, 2 (2004), p. 31-65.

Demoule, Jean-Paul, Landes, Christian (dir.), La fabrique de l'archéologie en France, Paris, La Découverte, 2009.

Díaz-Andreu, Margarita, A World History of Nineteenth-Century Archaeology: Nationalism, Colonialism, and the Past, Oxford, Oxford University Press, 2007.

Díaz-Andreu, Margarita, Champion, Timothy (eds), Nationalism and Archaeology in Europe, London, University College London Press, 1996.

Dietrer, Michael, «L’Archéologie du colonialisme. Consommation, emmêlement culturel et rencontres coloniales en Méditerranée", in Regards croisés sur le métissage, éd. par Laurier Turgeon, Québec, Presses de l'Université Laval, 2002, p. 135-184.

-, Archaeologies of Colonialism: Consumption, Entanglement, and Violence in Ancient Mediterranean France, Berkeley, University of California Press, 2010. 
Duluce, Sophie, Zytnicki, Colette (dir.), Décoloniser l'histoire?: de "l'histoire coloniale" aux histoires nationales en Amérique latine et en Afrique (XIX ${ }^{e}-X X^{e}$ siècles), Saint-Denis, Société française d'histoire d'outre-mer, 2003.

DulucQ, Sophie, Klein, Jean-François, Stora, Benjamin (éds), Les mots de la colonisation, Toulouse, Presses universitaires du Mirail, 2008.

Eschment, Beate, Harder, Hans (eds), Looking at the Coloniser. CrossCultural Perceptions in Central Asia and the Caucasus, Bengal, and Related Areas, Würzburg, Ergon Verlag, 2004 (Mitteilungen zur Sozial- und Kulturgeschichte der islamischen Welt 14).

Fenet, Annick, Documents d'archéologie militante: la mission Foucher en Afghanistan (1922-1925), Paris, De Boccard, 2010 (Mémoires de l'Académie des inscriptions et belles-lettres 42).

FORÊT, Philippe, La véritable histoire d'une montagne plus grande que l'Himalaya: les résultats scientifiques inattendus d'un voyage au Tibet (1906-1908) et de la querelle du Transhimalaya, Paris, Bréal, 2004.

Galuzo, Pjotr Grigor'evič, Turkestan - kolonija [Le Turkestan - une colonie], Moskva, Izdatel'stvo kommunističeskogo universiteta trudjaščikhsja Vostoka, 1929.

Geraci, Robert P., Window on the East: National and Imperial Identity in Late Tsarist Russia, Ithaca, Cornell University Press, 2001.

Geyer, Dietrich, "Modern Imperialism? The Tsarist and the Soviet Examples", in Imperialism and After. Continuities and Discontinuities, ed. by Wolfgang Justin Mommsen, Jürgen Osterhammel, London, Allen \& Unwin, 1986, 49-62.

Gillot, Laurence, La mise en valeur des sites archéologiques: un rapprochement entre Archéologie, Tourisme et Développement. Le cas de la Syrie, 2 vols, thèse de doctorat, Université Libre de Bruxelles, 2008 (non publié).

Given, Michael, The Archaeology of the Colonized, London/New York, Routledge, 2004.

Gutron Clémentine, L'archéologie en Tunisie (XIX'e-XXe siècles). Jeux généalogiques sur l'Antiquité, Paris, Karthala Editions, 2010.

Gorshenina, Svetlana, «Premiers pas des archéologues russes et français dans le Turkestan russe (1870-1890): méthodes de recherche 
et destin des collections", Cahiers du Monde Russe, 40/3 (1999), p. 365-384.

-, Explorateurs en Asie centrale. Voyageurs et aventuriers de Marco Polo à Ella Maillart, Genève, Olizane, 2003.

-, Private Collections of Russian Turkestan in the 2nd Half of the 19th and Early 20th Century, Berlin, Klaus Schwarz Verlag, 2004 (ANOR15, Institut für Orientalistik, Halle, Mittelasienwissenschaft Humboldt-Universität, Berlin, Université de Lausanne).

—, «De l'archéologie touristique à l'archéologie scientifique. L'archéologie en Asie centrale de la conquête russe du Turkestan à l'aube de l'époque soviétique: la "non-archéologie" occidentale", in ISIMU-6. El Redescubrimiento del Asia Central: nuevos horizontes en la historia y la arqueologia del Oriente antiguo, Madrid, Universidad Autonoma de Madrid, 2006, p. 33-46 (Cuadernos del seminario Walter Andrae, año Académico 2003-2004, VI, I).

_, «La marginalité du Turkestan colonial russe est-elle une fatalité, ou l'Asie centrale postsoviétique entrera-t-elle dans le champ des Post-Studies", in Le Turkestan russe: une colonie comme les autres?, Cahiers d'Asie centrale 17-18, éd. par Svetlana Gorshenina, Sergej Abashin, Paris, Complexe/IFEAC, 2009, p. 17-76.

—, "La construction d'une image "savante" du Turkestan russe lors des premières expositions "coloniales" dans l'empire: analyse d'une technologie culturelle du pouvoir", in Le Turkestan russe: une colonie comme les autres?, Cabiers d'Asie centrale 17-18, éd. par Svetlana Gorshenina, Sergej Abashin, Paris, Complexe/IFEAC, 2009, p. 133-178.

—, «Le Recueil Turkestanais de Mežov. L'utopie d'une somme exhaustive des connaissances sur l'Asie centrale", Asiatische Studien - Etudes Asiatiques, LXV/3 (2011), p. 625-660.

-, Asie centrale. L'invention des frontières et l'héritage russo-soviétique, Paris, CNRS-Editions, 2012.

—, «Voyage de Léon de Beylié à Samarkand en été 1888: une lecture à travers des idées reçues", in L'empire d'un officier. Le général Léon de Beylié: mise en perspective. Actes du colloque du Musée de Grenoble janvier 2011, éd. par Jean-François Klein, à paraître.

Gorshenina, Svetlana, Aвashin, Sergej (éds), Le Turkestan russe. Une colonie comme les autres?, Cahiers d'Asie centrale 17-18, Paris, Complexe/IFEAC, 2009. 
Gorshenina, Svetlana, Rapin, Claude, Les archéologues en Asie centrale: de Kaboul à Samarcande, Paris, Gallimard, 2001 (Découvertes Gallimard 411).

—, «Kak istorik i arkheolog prevratilis’ v buržuaznykh propagandistov. Otvet na recenziju G. A. Košelenko » ["Comment une historienne et un archéologue se sont mués en propagandistes bourgeois. Réponse à un compte rendu de G. A. Košelenko»], Kul'turnye cennosti - Cultural Values: 2002-2003, Bibliotheca Turkmennica, Sankt-Peterburg, European House, 2004, p. 191-204 (version complète); Idem, in Rossijskaja arkheologija, 2 (2005) p. 102-109 (version abrégée). Version en ligne: http://svetlana.gorshenina. free.fr/1ARCH30_RusArkh_Koshel.pdf

—, «De l'archéologie russo-soviétique en situation coloniale à l'archéologie post-coloniale en Asie centrale", in Archéologie(s) en situation coloniale: paradigmes et situations comparées, Les Nouvelles de l'archéologie 126, éd. par Alexandra Galitzine-Loumpet, Svetlana Gorshenina, Claude Rapin, Paris, Maison des sciences de l'homme/Errance, 20II, vol. 1, p. 29-33.

Gosden, Chris, Archaeology and Colonialism: Cultural Contact from 5000 B. C. to the Present, Cambridge/New York, Cambridge University Press, 2004.

Gran-Aymerich, Eve, "L'archéologie française à l'étranger. Méditerranée, Afrique et Proche-Orient (1945-1970). Vers un nouvel équilibre», La Revue pour l'histoire du CNRS [en ligne], 5 (2001) : http://histoire-cnrs.revues.org/3402 ?\&id=3402

Guha-Thakurta, Tapati, "Production et reproduction d'un monument: le stupa de Sanchi dans l'Inde coloniale», Annales HSS, 6 (2010), p. 1403-1432.

Hauner, Milan, What is Asia to us? Russia's Asian Heartland Yesterday and Today, London/New York, Routledge, 1992.

Hirsch, Francine, Empire of Nations: Ethnographic Knowledge and the Making of the Soviet Union, Ithaca, Cornell University Press, 2005.

Hobsbawm, Eric, Ranger, Terence, The Invention of Tradition, Cambridge, Cambridge University Press, 1983.

Hopкirк, Peter, Foreign Devils on the Silk Road: the Search For the Lost Cities and Treasures of Chinese Central Asia, London, J. Murray, 1980. 
IRW IN, Robert, For Lust of Knowing. The Orientalists and their Enemies, London/New York, Allen Lane The Penguin Press, 2006.

Jersild, Austin, Orientalism and Empire: North Caucasus Mountain Peoples and the Georgian Frontier, 1845-1917, Montreal, McGillQueen's University Press, 2002.

Kaeser, Marc-Antoine, "Nationalisme et archéologie: quelle histoire?", Revue d'Histoire des Sciences Humaines, 2 (2000), p. 155-163.

Kelly, Kenneth G., Hardy, Meredith D. (eds), French Colonial Archaeology in the Southeast and Caribbean, Gainesville, University Press of Florida, 2011.

Kennedy, Dane, "Imperial History and Post-Colonial Theory», The Journal of Imperial and Commonwealth History, 24/3 (1996), p. 345-363.

Khalid, Adeeb, "Between Empire and Revolution. New Work on Soviet Central Asia", Kritika: Explorations in Russian and Eurasian History, 7/4 (2006), p. 865-884.

—, "Backwardness and the Quest for Civilization: Early Soviet Central Asia in Comparative Perspective», Slavic Review, 65/3 (2006), p. 231-251.

—, "Locating the (Post-)Colonial in Soviet History", Central Asian Survey, 26/4 (2007), p. 465-473.

Kimitaka, Matsuzato (ed.), Imperiology: From Empirical Knowledge to Discussing the Russian Empire, Sapporo, Slavic Research Center, Hokkaido University, 2007.

Klein, Jean-François, Suremain, Marie-Albane de, "Clio et les colonies. Retour sur des historiographies en situation", Romantisme, 139 (2008), p. 59-80.

Kohl, Philip L., Fawcett, Clare (eds), Nationalism, Politics, and the Practice of Archaeology, Cambridge, Cambridge University Press, 1995.

Kuropatkin, Aleksej, "Zapiska zavedujuščego Aziatskoj čast’ju glavnogo štaba polkovnika A. N. Kuropatkina po afganskomu voprosu, 27 nojabrja 1878 g. » [«Note du responsable du département asiatique de l'état-major du colonel A. N. Kuropatkin sur la question afghane du 27 novembre 1878 », in "Bol'saja igra»v Central'noj Azii: "Indijskij pokhod» russkoj armii. Sbornik arkhivnykh dokumentov ["Le Grand Jeu" en Asie centrale: "l'expédition 
indienne" de l'armée russe. Recueil de documents d'archives], Moskva, Institut Vostokovedenija RAN, 2005 [1878], p. 207-208. LARUElLE, Marlène, L'Idéologie eurasiste russe ou comment penser l'empire, Paris, L'Harmattan, 1999.

—, Mythe aryen et rêve impérial dans la Russie du XIXe siècle, Paris, CNRS-Editions, 2005.

—, "Le paradigme du colonialisme en Asie centrale postsoviétique», L'Homme et la Société, 174 (2009), p. 27-40.

—, «Le berceau aryen: mythologie et idéologie au service de la colonisation du Turkestan", in Le Turkestan russe: une colonie comme les autres?, Cahiers d'Asie centrale 17-18, éd. par Svetlana Gorshenina, Sergej Abashin, Paris, Complexe/IFEAC, 2009, p. 107-131.

Legendre, Jean-Pierre, Olivier, Laurent, Schnitzler, Bernadette (éds), L'archéologie nationale-socialiste dans les pays occupés à l'ouest du Reich. Actes de la table ronde internationale "Blut und Boden" tenue à Lyon (Rhône) dans le cadre du Xe congrès de l'European Association of Archaeologists (EAA), les 8 et 9 septembre 2004, Gollion, Infolio, 2007.

Litvinskij, Boris Anatol'evič (otv. red.), «Zakaspijskij kružok ljubitelej arkheologii i istorii» [ "Le cercle transcaspien des amateurs d'archéologie et d'histoire»], Izvestija otdelenija obščestvennykh nauk Tadž. SSR, Dušanbe, 14 (1957), p. 157-167.

-, Vostočnyj Turkestan $v$ drevnosti i rannem srednevekov'e [Le Turkestan oriental dans l'Antiquité et au haut Moyen Age], Moscou, Vostočnaja literatura, 2000.

Lyons, Claire L., Papadopoulos, John K., The Archaeology of Colonialism, Los Angeles, The Getty Research Institute, 2002.

Maffi, Irène, Pratiques du patrimoine et politiques de la mémoire en Jordanie: entre histoire dynastique et récits communautaires, Lausanne, Ed. Payot, 2004.

Martin, Terry Dean, The Affirmative Action Empire: Nations and Nationalism in the Soviet Union, 1923-1939, Ithaca, Cornell University Press, 2001.

Masson, Mikhail Evgen'evič, "Kratkij očerk istorii izučenija Srednej Azii v arkheologičeskom otnošenii» ["Court traité d'histoire de l'étude de l'Asie Centrale d'un point de vue archéologique»], in Trudy Sredneaziatskogo gosudarstvennogo universiteta. Novaja serija 
[CEuvres de l'Université d'Etat de l'Asie centrale], Taškent, 1956, vyp. $80, \mathrm{n}^{\mathrm{o}} 12$.

Meaux, Lorraine de, La Russie et la tentation de l'Orient, Paris, Fayard, 2010.

MÉjow, Vladimir, Recueil du Turkestan comportant des livres et des articles sur l'Asie centrale en général et la province du Turkestan en particulier. Indicateur systématique et alphabétique, Sankt-Peterburg, 1878.

Moro-Aвadía, Oscar, "The History of Archaeology as a "Colonial Discourse" ", Bulletin of the History of Archaeology, 16/2 (2006), p. 4-17.

Morrison, Alexander S., Russian Rule in Samarkand 1868-1910. A Comparison with British India, Oxford, Oxford University Press, 2008.

Nanta, Arnaud, Débats sur les origines du peuplement de l'archipel japonais dans l'anthropologie et l'archéologie (décennie 1870-décennie 1990), thèse de doctorat sous la direction de A. Horiuchi et C. Liauzu, Université Paris 7, 2004.

Nordman, Daniel, Raison, Jean-Pierre (éds), Sciences de l'homme et conquête coloniale. Constitution et usages des sciences humaines en Afrique (XIX ${ }^{e}-X X^{e}$ siècles), Paris, Presses de l'Ecole normale supérieure, 1980.

Olivier-Utard, Françoise, Politique et archéologie. Histoire de la Délégation archéologique française en Afghanistan (1922-1982), Paris, Ed. Recherche sur les civilisations, 1997.

Oulebsir, Nabila, Les usages du patrimoine: monuments, musées et politique coloniale en Algérie (1830-1930), Paris, Editions de la Maison des Sciences de l'Homme, 2004.

PAI, Hyung Il, Constructing "Korean" Origins. A Critical Review of Archaeology, Historiography, and Racial Myth in Korean State Formation Theories, Cambridge Mass., Harvard University Asia Center, 2000.

Petitjean, Patrick (éd.), Les sciences hors d'Occident au XXe siècle. Vol. 2, Les sciences coloniales, figures et institutions $=20$ th Century Sciences: Beyond the Metropolis. Vol. 2, Colonial Sciences: Researchers and Institution, Paris, Orstom, 1996.

Petrov, Andrej Evgen'evič, Šnirel'man, Viktor Aleksandrovič, Falsifikacija istoričeskikh istočnikov i konstruirovanie 
ètnokratičeskikh mifov [Falsification des sources historiques et construction des mythes ethnocratiques], Moscou, IA RAN, 2011.

Pianciola, Niccolò, «Décoloniser l'Asie centrale? Bolcheviks et colons au Semirech'e (1920-1922)", Cahiers du monde russe, 49/1 (2008), p. 101-144.

Popova, Irina Fedorovna, "Russian Expeditions to Central Asia at the Turn of the 20th Century", in Russian Expeditions to Central Asia at the Turn of the 20th Century, ed. by Irina Fedorovna Popova, Sankt-Peterburg, Slavia Publishers, 2008, p. 11-39.

Pouchepadass, Jacques, "Que reste-t-il des Subaltern Studies?", Critique internationale, 3/24 (2004), p. 67-79.

—, "Le projet critique des postcolonial studies entre hier et demain", in La situation postcoloniale. Les "postcolonial studies" dans le débat français, éd. par Marie-Claude Smouts, Paris, Presses de Sciences PO, 2007, p. 173-218.

Ray, Himanshu Prabha, Colonial Archaeology in South Asia (18611948): The Legacy of Sir Mortimer Wheeler, New Delhi, Oxford University Press, 2007.

Rieber, Alfred J., Miller, Alexei (eds), Imperial Rule, Budapest, Central European University Press, 2004.

Roitman, Janet (ed.), Racial France, Public Culture 23/1, 2011.

SAada, Emmanuelle (éd.), Regards croisés: Transatlantic Perspectives on the Colonial Situation, French Politics, Culture and Society 20/2, 2002.

Sahadeo, Jeff, Russian Colonial Society in Tashkent, 1865-1923, Bloomington, Indiana University Press, 2007.

SAÏD, Edward W., Orientalism, New York, Pantheon Books, 1978.

—, Culture and Imperialism, New York, A. A. Knopf, 1993.

SAnd, Christophe, Bole, Jacques, Ouetcho, André-John, «Evolutions $\mathrm{du}$ discours archéologique sur 150 ans d'histoire coloniale et postcoloniale en Nouvelle-Calédonie: un cas d'école ", in Archéologie(s) en situation coloniale: paradigmes et situations comparées, Les Nouvelles de l'archéologie 126, éd. par Alexandra Galitzine-Loumpet, Svetlana Gorshenina, Claude Rapin, Paris, Maison des sciences de l'homme/Errance, 2011, vol. 1, p. 37-41.

Schimmelpenninck van der Oye, David, "Vasilij V. Vereshchagin's Canvases of Central Asian Conquest", in Le Turkestan russe: une colonie comme les autres?, Cahiers d'Asie centrale 17-18, éd. par 
Svetlana Gorshenina, Sergej Abashin, Paris, Complexe/IFEAC, 2009, p. 179-209.

-, Russian Orientalism. Asia in the Russian mind from Peter the Great to the emigration, New Haven, Yale University Press, 2010.

Schlanger, Nathan, «Identités recomposées: archéologie coloniale et identité nationale en Afrique du Sud", Les Nouvelles de l'Archéologie, 90 (2002), p. 20-22.

—, "Archéologie coloniale et identité nationale en Afrique australe hier et aujourd'hui", Cahier des thèmes transversaux ArScAn, Thème 5: Culture et Identité, IV (2002/2003), s. p.

Shepherd, Nick, "Heading South, Looking North. Why we Need a Post-Colonial Archaeology", Archaeological Dialogues, 9/2 (2002), p. 74-82.

Sibeud, Emmanuelle, Une science impériale pour l'Afrique? La construction des savoirs africanistes en France 1878-1930, Paris, Ed. de l'EHESS, 2002.

—, "Les sciences sociales à l'épreuve de la situation coloniale", Revue d'Histoire des Sciences Humaines, 1/10 (2004), p. 3-7.

—, «Post-Colonial et Colonial Studies: enjeux et débats», Revue d'histoire moderne et contemporaine, 5/51-4bis (2004), p. 87-95.

Silliman, Stephen W., "Culture Contact or Colonialism? Challenges in the Archaeology of Native North America", American Antiquity, 70/1 (2005), p. 55-74.

Simoni, Ilaria, Archeologia coloniale in Libia (1911-1943). Propaganda e Ricerca storica, thèse de doctorat sous la dir. du Prof. Maurizio Paoletti, Université de Pise, 2006.

Singaravélou, Pierre, "Le moment "impérial” de l'histoire des sciences sociales (1880-1910)", Mille neuf cent. Revue d'histoire intellectuelle, 27 (2009), p. 87-102.

Slezkine, Yuri, Arctic Mirrors: Russia and the small peoples of the North, Ithaca/London, Cornell University Press, 1994.

Smouts, Marie-Claude (éd.), La situation postcoloniale. Les "postcolonial studies" dans le débat français, Paris, Presses de Sciences PO, 2007.

Solov'Jov, Viktor Stepanovič, U istokov arkheologii Tadžikistana (18131917) [Aux sources de l'archéologie du Tadjikistan (1813-1917)], Elec, Izdatel'stvo Eleckogo universiteta, 2005. 
Sunderland, Willard, Taming the Wild Field, Colonization and Empire on the Russian Steppe, Ithaca/London, Cornell University Press, 2006.

—, "What is Asia to Us? Scholarship on the Tsarist "East" since the 1990s", Kritika: Explorations in Russian and Eurasian History, 12/4 (2011), p. 817-834.

Suvorova, Natal'ja Gennad'evna (otv. red.), Aziatskaja Rossija: ljudi i struktury imperii [La Russie d'Asie: les hommes et les structures de l'empire], Omsk, Nacional'nyj universitet Omska, 2005.

Thiesse, Anne-Marie, La création des identités nationales: Europe XVIII ${ }^{e_{-}}$ $X X^{e}$ siècles, Paris, Seuil, 2001.

Tolz, Vera, "Russia's own Orient»: The Politics of Identity and Oriental Studies in Late Imperial and Early Soviet periods, Oxford, Oxford University Press, 2011.

TRIGger, Bruce G., "Alternative archaeologies: nationalist, colonialist, imperialist", Man, 19/3 (1984), p. 355-370.

TrüMPler, Charlotte (Hrsg.), Das grosse Spiel: Archäologie und Politik zur Zeit des Kolonialismus (1860-1940). Begleitbuch zur Ausstellung Das Große Spiel - Archäologie und Politik, Ruhr-Museum, Weltkulturerbe Zollverein, Essen, 11. Februar-13. Juni 2010, Köln, DuMont, 2008.

Ujfalvy de Mezö-Kövesd, Charles-Eugène de, Le Syr-Daria. Le Zérafchane. Le pays des sept-rivières et la Sibérie occidentale, Paris, éd. Ernest Leroux, 1887.

Uкнтомккіј, Èsper Èsperovič, $K$ sobytijam v Kitae. Ob otnošenijakh Zapada i Rossii k Vostoku [Sur les événements survenus en Chine. Sur les relations de l'Occident et de la Russie avec l'Orient], SanktPeterburg, Vostok, 1900.

Uyama, Tomohiko (ed.), Empire, Islam, and Politics in Central Eurasia, Sapporo, Slavic Research Center, Hokkaido University, 2007.

Vertukhov, Dmitrij, "Kolonial'naja arkheologija» ["Archéologie coloniale»], http://www.plato-ukok.info/text/240 (consulté le 11 juillet 2011).

Volait, Mercedes, Fous du Caire: excentriques, architectes et amateurs d'art en Egypte (1867-1914), Forcalquier, L'Archange Minotaure, 2009. 
Waselkov, Gregory, The Archaeology of French Colonial North America, Tucson, AZ, Society for Historical Archaeology, 1997 (Guide to Historical Archaeological Literature 5).

Zelenev, Evgenij Il'ič, Kasevič, Vadim Borisovič, Vvedenie v vostokovedenie [Introduction à l'orientalisme], Sankt-Peterburg, Karo, 2010.

Crédits iconographiques

Fig. 1:

Archives de l'auteur.

Fig. 2:

Tiré de: Turkestanskij sbornik, t. 504, p. 182a.

Fig. 3:

Tiré de: Turkestanskij sbornik, t. 413, p. 21. 
